\title{
(4) \\ FY05 LDRD Final Report \\ A Computational Design Tool for Microdevices and Components Used in Pathogen Detection Systems \\ D. Trebotich
}

February 10, 2006 


\section{Disclaimer}

This document was prepared as an account of work sponsored by an agency of the United States Government. Neither the United States Government nor the University of California nor any of their employees, makes any warranty, express or implied, or assumes any legal liability or responsibility for the accuracy, completeness, or usefulness of any information, apparatus, product, or process disclosed, or represents that its use would not infringe privately owned rights. Reference herein to any specific commercial product, process, or service by trade name, trademark, manufacturer, or otherwise, does not necessarily constitute or imply its endorsement, recommendation, or favoring by the United States Government or the University of California. The views and opinions of authors expressed herein do not necessarily state or reflect those of the United States Government or the University of California, and shall not be used for advertising or product endorsement purposes.

\section{Auspices Statement}

This work was performed under the auspices of the $U$. S. Department of Energy (DOE) by the University of California, Lawrence Livermore National Laboratory (LLNL) under Contract No. W-7405-Eng-48. The project (03-ERI-003) was funded by the Laboratory Directed Research and Development Program at LLNL. 


\title{
FY05 LDRD Final Report A Computational Design Tool for Microdevices and Components Used in Pathogen Detection Systems LDRD Project Tracking Code: 03-ERI-003 David Trebotich, Principal I nvestigator
}

\begin{abstract}
We have developed new algorithms to model complex biological flows in integrated biodetection microdevice components. The proposed work is important because the design strategy for the next-generation Autonomous Pathogen Detection System at LLNL is the microfluidic-based Biobriefcase, being developed under the Chemical and Biological Countermeasures Program in the Homeland Security Organization. This miniaturization strategy introduces a new flow regime to systems where biological flow is already complex and not well understood. Also, design and fabrication of MEMS devices is time-consuming and costly due to the current trial-and-error approach. Furthermore, existing devices, in general, are not optimized. There are several MEMS CAD capabilities currently available, but their computational fluid dynamics modeling capabilities are rudimentary at best. Therefore, we proposed a collaboration to develop computational tools at LLNL which will (1) provide critical understanding of the fundamental flow physics involved in bioMEMS devices, (2) shorten the design and fabrication process, and thus reduce costs, (3) optimize current prototypes and (4) provide a prediction capability for the design of new, more advanced microfluidic systems. Computational expertise was provided by Comp-CASC and UC Davis-DAS. The simulation work was supported by key experiments for guidance and validation at UC Berkeley-BioE.
\end{abstract}

\section{I ntroduction/ Background}

BioMEMS devices. Micro Electro Mechanical Systems, or MEMS, devices are ideal for the biodetection effort because they are small, low-powered and distributable. New biological and chemical pathogen detection systems, or "bio-smoke alarms" such as the Biobriefcase at LLNL, will consist of several microfluidic components in a MEMS-based system with functions including polymerase chain reaction and immunoassay (see Figure 1). This new design strategy improves the current Autonomous Pathogen Detection System through lower reagent consumption, lower operating and manufacturing costs, decreased reaction times, networkability and the ability to mass-distribute these miniaturized systems in a public setting.

MEMS technology has advanced over the last few years but for the most part the resulting devices are early generation prototypes, generally of individual components cobbled together, that demonstrate proof-of-principle like the drug delivery system in Figure 2a. Significant work remains to create integrated working devices like the micro-mixer in Figure $2 \mathrm{~b}$, and still more is required to optimize at the system level. The research and development needed for advances is costly and slow, however, because microfabrication and testing of devices can take on the order of months per cycle. Current design strategies are ultimately trial-and-error. Therefore, accurate CAD tools are needed to shorten the design/fabrication cycle of these devices. 
There are several MEMS CAD capabilities currently available that have evolved from industry design tools for integrated circuits coupled with mechanical simulation capabilities. Furthermore, many, if not all, biosensors will have significant fluidic components because of the biochemical nature of the assays. While some of the CAD packages have rudimentary fluid dynamics modeling capabilities, for advanced design and optimization of biodetection systems it will be necessary to develop an advanced modeling capability to address and understand the fundamental physics and chemistry associated with complex biological flow in micro-environments.

Complex biological fluids at the microscale. One of the fundamental research problems in this project is polymeric fluid dynamics at the microscale. The biochemical nature of biosensing and detection requires micro-processing of macromolecules (polymers) such as DNA or proteins via polymerase chain reaction (PCR) or immunoassay, respectively. In a standard amplification and sequencing microprocessor $10^{10}$ molecules of bacterial DNA, each of which is approximately 1-2 $\mathrm{mm}$ long when stretched out in solution, will have to pass through channels with characteristic length scales of $100 \mu \mathrm{m}$ or less. This flow regime presents new fluid mechanical issues, different from macro-scale flows, because (1) surface-to-volume ratios are extremely large in microchannels; (2) biological fluids demonstrate complex, non-Newtonian behavior; and (3) characteristic lengths of the macromolecules/cells approach those of the fluid channels.

Modeling complex biological fluids is a challenge because their non-Newtonian constitutive behavior is not easily represented. For example, a highly concentrated solution of suspended polymer molecules such as DNA may be represented at large, system-level scales with a continuum viscoelastic constitutive model. This flow scenario is typical of a post-amplification process in a PCR device where it is computationally prohibitive to discretely represent every DNA molecule. However, when the inter-polymer spacing is comparable to geometry length scales as in a microscale flow, a continuum approximation is no longer appropriate. Instead, a discrete molecular approximation is needed, but at a computational expense. On the other hand, in a pre-amplification detection environment very few DNA molecules may be present in the flow as a result of some collected air sample that has been fluidized; downstream, only a single molecule needs to be captured for amplification. In this case a discrete molecular approximation is appropriate, but smaller scale chemistry must be included in the model for the capture process. Figures 3 and 4 demonstrate these continuum and molecular behaviors, respectively.

Figure 3 is experimental evidence obtained in this project (discussed in more detail later) that proves the hypothesis that the presence of long-chain polymers in a Newtonian solvent (e.g., Boger fluid, DNA) can cause a bulk fluid to demonstrate both viscous and elastic behavior even at micro-molar concentrations. This behavior is drastically different from canonical flows as seen in the vortex enhancement in reentrant corners. One potential problem scenario of this phenomenon occurring in a biosensor is the diffusion of a macromolecule across streamlines into one of these large recirculation zones, then degradation or even breaking of the molecule in the vortex due to shear and finally diffusion back across streamlines to be processed downstream without full character. It is important to be able to predict this type of behavior in a device where there is a need to control the working fluid and simultaneously manipulate and detect biological species in the fluid.

Figure 4 demonstrates the conformational changes of DNA molecules as they move through a micro-valve [1]. Experiments show that long-chain polymers migrate 
away from regions of high shear. Also, DNA molecules stretch out in accelerating flow regions, and recoil in decelerating flow or stagnant regions. These types of dynamics are relevant because they can affect assumed device performance in a biosensor, supporting the hypothesis that molecular-scale processes can have substantial system-level effects. Clearly, a design capability to predict the location of a DNA molecule, and when it can be transported through a microdevice without a change in character, would be a significant advantage over trial-and-error. More importantly, the ability to rationally design a bioMEMS device to predictably stretch out a DNA molecule on demand would have enormous impact on the development of advanced biosensors using single molecule identification.

\section{Research Activities}

We set out in this project to develop two approaches to modeling particle-laden fluids at the microscale. The first is a new continuum algorithm for modeling DNA in solution at the microscale as a viscoelastic fluid. The second is a particle representation of polymers in fluid to predict the fate of an individual DNA molecule in complex microdevice components. A benchmark or model problem was considered for each approach.

The High Weissenberg Number Problem. Since DNA is a long-chain polymer in solution, like a Boger fluid, we chose to model it at the continuum level with the Oldroyd-B constitutive equation. This is an acceptable scale to model from a device design perspective as bulk fluid velocities and pressure drops are the key parameters for flow control. Historically, Oldroyd-B fluids have suffered from the inability of numerical methods to compute solutions beyond a frustratingly low value of the Weissenberg number (elasticity) in 4 to 1 sudden contraction flows with solvent viscosity ratio of $1 / 9$, a benchmark problem known as the High Weissenberg Number Problem (HWNP) [8-12]. Computational symptoms of the problem include large normal stresses near geometric singularities and worsening of the problem with refinement. The critical high Weissenberg number value is $\mathrm{O}(1)$. Furthermore, axisymmetric contractions have received much attention in macroscale Oldroyd- $B$ flows because of the large recirculation zones that develop in the salient corner of the contraction [13]. Our focus was on planar contractions because of MEMS fabrication limitations, and microscale flows; vortex enhancement in the corner is less intense at the macroscale [14, 15].

DNA extraction model problem. As a test problem for particle-fluid coupling we focused our effort on modeling DNA extraction in a polymerase chain reaction (PCR) chamber as part of the Biobriefcase microfluidic-based pathogen detection system. The primary extraction technique currently being pursued by our device collaborators at LLNL is a packed bed reactor (Figure 13a), which is essentially a small tube packed with approximately $50 \mu \mathrm{m}$-diameter glass beads. The physical model presents a 3D multi-scale problem where DNA molecules must be resolved along with the flow geometry. A second PCR extraction design is a pillar chip which is an array of cylindrical obstructions in a shallow microchannel (Figure 13c). Though a $3 \mathrm{D}$ problem this latter design lends itself to $2 \mathrm{D}$ models. The restriction to $2 \mathrm{D}$ is useful and can be reduced to model a single molecule traveling through a smaller section of the array. This is the model problem where we honed our algorithm development to model relevant physics and chemistry for full 3D problems.

This model problem offers possibilities for processing polymer-laden fluids. For example, when DNA-laden fluids flow through an array of pillars, size separation may 
be achieved: longer molecules are slowed by their interaction with the pillars to a greater degree than are smaller molecules. Pillar rods may also be electrochemically treated to promote selective binding of macromolecules, yielding a mechanism for "sticking". Underlying flow fields in the vicinity of complex geometry variations, as in a pillar array or abrupt contraction, display steep velocity gradients. The shear forces due to these gradients can be strong enough to break molecular bonds, a dynamic which can be useful in a PCR device but detrimental in a drug delivery system. Another possibility for this model is design of a geometric configuration such as a contraction into a very narrow channel through which a polymer chain is " threaded" for single molecule detection or even sequencing. The success of modeling such strategies relies critically on the fluid-molecule coupling that occurs in microdevice channels.

\section{Technical Approach}

Our work required advanced algorithmic features in order to treat the threedimensional, multi-scale, multi-species, multi-physics problems encountered in the DNA extraction model problem. We rely upon high-resolution finite difference methods for the discretization of Newtonian and non-Newtonian fluids. The continuum method is tightly coupled to a new particle representation with reverse coupling, including chemistry and electrokinetics. We use a volume of fluid approach to treat irregular geometries. The model is built on a software framework which contains the support for adaptivity, complex geometry and scalability needed for large scale simulation.

Fluid discretization. In our fluid continuum approach we solve the incompressible Navier-Stokes equations which will include a generic source term on the right-hand side of the momentum equation. With this source term we couple the fluid to nonNewtonian stress models such as the Oldroyd-B constitutive relation for the extra stress involved in polymeric flows [16] and others in a modular fashion. It is through this source term that we also couple the particle representation. Our underlying spatial discretization for these problems is high-resolution finite-difference methods (typically, second-order accurate in space). Our time discretization is the semi-implicit predictor-corrector approach of Bell, Colella and Glaz (BCG) $[17,18]$. When applied to systems such as incompressible viscoelastic flow or advectiondiffusion equations, there are hyperbolic terms, parabolic viscous terms, and an elliptic divergence-free constraint. Under these circumstances, one obtains methods that are second-order accurate in space and time, have a time-step constraint based on the advective time scale only (CFL condition), and involve the solution of very well-behaved linear systems, i.e., ones coming from standard discretizations of second-order elliptic and parabolic operators. This allows us to employ methods for solving these systems based on multigrid, that is, fast linear solver, concepts.

Particle representation. In order to track a molecule like DNA, we couple a particle method to the continuum model using a PIC, or particle-in-cell representation. This is based on an approach (e.g., [19]) that represents the physical dynamics of large molecules as a collection of point masses "connected" with spring-like forces. Fluid-molecule interactions, approximated as Stokes drag with a stochastic thermal (Brownian) component, affect both the particle's Newtonian time evolution, and act also to change the fluid's dynamics. This approach to particle-fluid coupling distinguishes our strategy from those of other workers, who consider the fate of biomolecules in prescribed flow fields. At high polymer concentration, and in channels with molecular length scales, the full coupling is 
important and can be uniquely addressed by our method. The particle-fluid interaction is short-ranged, so many complications of the PIC methodology (as used in plasma physics, for example) do not apply here. The basic notion of grid-to-point local mappings and inverse mappings is used. The only long-range interaction is the incompressibility constraint, a divergence-free condition on the velocity field which, via the momentum equations, requires the particle-fluid interaction force to be divergence-free as well. Strategies for accelerating the convergence of this method are suggested in [20].

Volume of fluid/ embedded boundary methods. In order to treat complex geometries we use a volume-of-fluid representation of the discretized solution near the boundary [21-23]. This technique is also known as the embedded boundary method. In this approach, the surface is represented by its intersection with an underlying rectangular grid, or a cookie-cutter approach. This leads to a natural, finite-volume discretization of the PDE on irregular control volumes adjacent to the boundary. However, the primary unknowns are assumed to live at the centers of the Cartesian grid control volumes, i.e., as if the boundary wasn't there. Such an approach has been shown to give consistent and stable discretizations, even in the case of moving boundaries [24]. It is also routine to prescribe fluxes through the irregular boundaries using this approach. One of the principal advantages of the embedded boundary method is that the problem of generating the description of the geometry on the grid starting from surface tessellations produced, for example, by a CAD system has been completely solved [25].

Our complex geometry approach using embedded boundaries is complementary to a fast and accurate technique to extract surface data from medical images without loss in geometric detail $[3,4]$. Anatomical surfaces are extracted by means of level-sets methods that accurately model the complex and varying surfaces of pathological objects such as aneurysms and stenoses. The surfaces obtained are defined at the sub-pixel level where they intersect the Cartesian grid of the image domain. It is therefore straightforward to construct embedded boundary representations of these objects on the same grid. While most classical techniques require construction of a structured mesh that approximates the surface in order to extrapolate a 3D finiteelement gridding of the whole volume, our method directly simulates the flow inside the extracted surface without losing any complicated details and without building additional grids. This feature makes the embedded boundary method superior to body-fitted unstructured grids used for finite element simulations. With the embedded boundary method meshing is more tractable and less expensive computationally, especially in the case of a moving boundary as re-gridding is unnecessary, requiring only a conservative movement of the boundary.

Electrokinetics. The working fluid in our models is typically driven by pressure. However, microfluidic devices also use a voltage drop, and thus an electric field, to drive flow. The origin of electrokinetic phenomena is the interaction of an applied electric field with the thin electrically charged layer that forms at fluid-solid interfaces. In this interfacial region, the electric field induces a body force in the charged fluid which drives fluid flow. However, because the electrically charged region of the fluid, often referred to as the diffuse-charge or Debye screening layer, is very thin (typically only 1 to $100 \mathrm{~nm}$ thick), the coupling between the electric field and the fluid flow only shows up in the mathematical formulation as a slip-velocity boundary condition. The boundary conditions for the electrostatics problem can be a bit more complicated depending on the material properties of the solid phase and the dynamics of diffuse-layer charging [26]. For the purposes of this project, we have 
assumed that the solid phase is nonpolarizable and that the characteristic time-scale of diffuse-layer charging is very small relative to the characteristic time-scale of the fluid flow. These approximations allow us to use a simple homogeneous Neumann boundary condition for the electrostatics problem. It is worth noting that the electrostatics problem is completely decoupled from the fluid flow. As a result, the full problem can be solved by first solving for the electric field and subsequently solving the Navier-Stokes equations using boundary conditions calculated from the solution to the electrostatics problem.

Software approach. We built our new physics algorithms on the Chombo structured-grid framework which is being developed at LBNL under the DOE Office of Science SciDAC program as part of the Applied Partial Differential Equations Integrated Software Infrastructure Center (APDEC ISIC). Chombo provides adaptive, embedded boundary/volume-of-fluid solvers and high performance, parallel support for all the major algorithmic components in this project. This includes elliptic solvers for incompressible flow based on multigrid, higher-order hyperbolic methods for advection and second-order time integrators. It is written in $\mathrm{C}++$ and FORTRAN. Releases are freely available to the public for download. The Chombo framework also contains an interface for receiving CAD and image descriptions of geometry and converting to embedded boundary Cartesian meshes.

\section{Results/ Technical Outcome}

We have developed two state-of-the-art numerical algorithms that have been used to simulate microscale biological flows in the two model problems.

Higher-order non-Newtonian continuum modeling and validation. The first is a fundamentally new approach to the numerical modeling of viscoelastic continuum flow in an effort to model semi-dilute and concentrated solutions of DNA in contraction channel flows [27-29]. Our first approach to discretization of the viscoelastic equations of motion (incompressible Navier-Stokes equations, modified to include a polymeric contribution to the deviatoric stress, plus the Oldroyd-B constitutive relation for the polymer stress) was a semi-implicit method based on Lax-Wendroff $[27,29]$, with a projection method to enforce incompressibility [17, 18] and a continuous splitting of the viscoelastic terms using Duhamel's formula [29]. The algorithm is convergent and stable for a single CFL condition for the full range of elastic flows, including the benchmark " high Weissenberg number" problem of steady-state viscoelastic flow in 4:1 abrupt contractions, where all other methods have failed for the past 30 years. With this method we were able to perform preliminary validation studies involving low concentration solutions (Figure 5). A graduate student in our group at UCD and LLNL, Andy Nonaka, has been working on a higher-order approach to this algorithm. He has also performed the simulations for further validation studies of low concentration solutions. These studies have compared results from the viscoelastic flow algorithm with velocity fields and pressure drops from DNA experiments performed by another graduate student in our group, at UCB, Shelly Gulati (Figure 6) [30]. Shelly has also recently demonstrated experimentally large recirculation zones in the flow of a semi-dilute DNA solution in the salient corners of planar contractions (see Figure 3). This is a 3D viscoelastic, microscale phenomenon. Large areas of recirculation and vortex enhancement have been observed previously at the macroscale in axisymmetric contractions for viscoelastic, non-shear-thinning solutions, but not planar contractions, which are required in microfluidic devices due to fabrication limitations. Our viscoelastic Oldroyd-B model has not captured this behavior, and, therefore, an 
improved constitutive relation may be needed (such as the addition of shear-thinning in the White-Metzner model).

The novel concept in this work is a continuous splitting of the viscoelastic stress terms using Duhamel's principle that partitions terms into explicitly-determined hyperbolic and implicitly-determined elliptic parts. This splitting improves the CFLlimited stable time step by orders of magnitude for some problems while retaining the viscous and elastic limiting behavior of the original PDE. Starting with linear analysis we determine the eigenvalues of the system and prove hyperbolicity (positivity of the stress) by introducing the inverse deformation gradient. We can then define a new wave speed from the quasi-linear advection form of the system and design to the viscous and elastic limits of the original PDE, while allowing some elastic freedom in recovering the viscous limit for well-posedness (see [29] for details). Oldroyd-B can then be re-written where the left-hand side is hyperbolic and well-posed, and the right-hand side contains proper and improper source terms.

Our algorithm is stable for a single CFL condition for the full range of elastic flows -from the viscous to the elastic limits -- including HWNP, where all other methods have failed for several decades. The algorithm also proved to be surprisingly robust in resolving elastic shear waves in an incompressible flow of a Maxwell fluid (Oldroyd-B with $\mu_{s}=0$ ). If viscosity is added to the Maxwell fluid then hybrid effects of elastic wave propagation and steady-state viscous behavior can be seen. If enough viscosity is added then the critical HWNP limit can be reached at critical elastic Mach number, $M a=1$, where $W e=1$ and $R e=1$. (This assumes that $M a$ is defined by the total viscosity, not just the polymer component as in [29].) We have shown that even though the fluid looks to be steady-state at the HWNP limit it, in fact, is not steady as there is underlying wave behavior occurring. We further note that in the elastic limit we required $\mu_{\mathrm{p}} / \lambda$ to be finite for well-posedness, i.e., to prevent degeneracy in the quasi-linear system.

One other result from this algorithm that is noteworthy is the presence of singular structure in the solution just downstream of the contraction corner. It has not been determined if this behavior is physical or a numerical artifact $[29,31]$, though some members of the non-Newtonian fluid mechanics community embraced this result in discussions during the XIVth International Workshop for Numerical Methods in NonNewtonian Fluids, in Santa Fe, NM, J une 2005 [32]. Coupled with our other finding of underlying wave propagation, we believe that refinement is needed to not only obtain solutions in the asymptotic regime of convergence but also to resolve the fast underlying waves, which may be one in the same problem.

Fluid-particle coupling algorithm. The second foundation of our computational effort is an approach for modeling " bead-rod" polymers whose dynamics are fully coupled to an incompressible viscous solvent $[6,7]$. In this algorithm, long chain polymers such as DNA are represented as a chain of nodes separated by fictitious rigid rods [33]. The nodes are subject to forces by the fluid--both viscous Stokes drag and stochastic (thermal, Brownian motion); and the solvent experiences equal and opposite body forces. It is in this sense of obeying Newton's third law of motion that we consider the dynamics to be "tightly coupled". Additionally, the polymer nodes may experience elastic collisions with domain boundaries. With this numerical algorithm, we have been able to simulate polymer-boundary interactions which occur in size-separation and extraction devices (Figure 7). This algorithm promises to demonstrate for the first time the effect of a semi-dilute or concentrated solution of discretized particles on a fluid. 
Particle interactions. In the freely-jointed ball-rod model we initially implemented, as with many other current implementations (e.g., [34]), crossing of rod sections is allowed. The resulting behavior has a strong theoretical foundation (e.g., [35]) and is therefore important for algorithm validation, but does not respect the correct noncrossing physical behavior of real molecules. Furthermore, macromolecules like DNA are charged, and chemically active. They interact through screened Coulombic interactions and migrate in response to imposed electric fields. Microfluidic separators have been designed based on the increase of residence time with molecule length in packed bed geometries, or through chemically-mediated residence time enhancement achieved by binding selective proteins to channel surfaces. These physical effects are characterized by intra-polymer, inter-polymer, and polymer-wall interaction potentials which may be long-ranged. We incorporated these Coulombic interactions into our model using a short-range Debye-Hückel potential. Results for intra-polymer and polymer-structure interactions are shown in Figure 8.

Electrokinetics. We addressed two test problems to verify our simulation capability for electro-osmotic flows: 2D flow in an empty rectangular channel with a DC electric field applied along the direction of the channel, and 2D flow around a circular post (with the same electro-osmotic mobility as the channel walls) in a rectangular channel with a DC electric field applied along the direction of the channel. For the first test problem, the full analytical solution is known for both the steady-state and time-dependent problems. For the steady problem, the velocity field is simply a plug flow determined from the parallel component of the electric field. For the timedependent problem, the velocity field is completely directed down the axis of the channel and only depends on the coordinate direction perpendicular to the channel. Figure 9 shows that the simulation is qualitatively correct; the slip-velocity at the boundary gradually diffuses into the interior of the channel and eventually reaches a steady-state which is a plug flow with the expected value of $0.1 \mathrm{~cm} / \mathrm{sec}$.

For the second problem, there is no analytical solution, but it is known that the steady-state solution is a potential flow that is proportional to the electric field with the constant of proportionality given by the electro-osmotic mobility. Figure 10 shows that near steady-state, the fluid velocity field produced by the simulation is in good agreement with this expectation. While the fluid has not quite yet reached steady-state, the similarity between the electric and velocity fields is evident. The value of the mobility computed by taking a few random points in the physical domain yields $b=7.81$ e- 04 which is getting close to the electro-osmotic mobility value of 0.001 prescribed at the boundaries. Figure 11 shows the time evolution of the fluid flow in response to an instantaneously applied electric field at $\mathrm{t}=0 \mathrm{sec}$.

We also applied the electrokinetic model to the staggered array geometry in the 2D fluid-particle model problem. We were not able to carry this computation out to a meaningful time due to a numerical instability encountered for low Reynolds number flows. This was unexpected as the semi-implicit approach for incompressible viscous flow should be stable for the advective CFL but also capture the viscous time scale. Figure 12 shows the electric field for this geometry. We continue to work this problem out using Stokes flow initialization techniques.

3D device simulation. Both numerical algorithms - continuum and hybrid -described above are robust and convergent for the model problems. We also have results for flows in more realistic, complicated geometries that model Biobriefcase 
designs (Figure 13a,c), including 3D (Figure 13b,d). One exemplary fluid dynamics question which the Biobriefcase project engineers sought to answer was: what is the largest operating flow rate for the device such that one DNA molecule is captured for amplification? We were able to address this question with our fluid-particle model. Initially, we found that low Reynolds number flows are dominated by the Brownian motion of the particles, inhibiting flow-through in an extraction chamber. On the other hand, the inertial effects in higher Reynolds number flows were too strong for polymer residence on a pillar. In the end we "validated" the operating Reynolds number of 1 for the flow-through device where viscous and inertial forces are essentially balanced, allowing a moderate residence time of a polymer molecule on an array structure for amplification.

\section{Coupling of embedded boundary method to medical image surface}

extraction. Our approach to complex geometry using embedded boundary methods has been leveraged to simulate cardio-pulmonary flows in realistic anatomies. We demonstrated this new approach to anatomical flows on a patient-specific carotid artery with stenosis (Figure 14) and a trachea which has undergone tracheostomy (Figure 15). The resolution of geometric detail in Figure 14 and 15 is a recent improvement over previous results in the field where surface meshes are a smooth representation of the original geometry, illuminating the advantage of our new approach over the previous state-of-the-art. Resolution of geometric details is important because small scale perturbations in the flow can have downstream effect as seen in the highly-resolved 2D results of Figures 16 and 17 . This capability can provide clinicians with time-sensitive and accurate information needed to evaluate how pathologies form, how they evolve, and ultimately how they are effectively treated.

\section{Exit Plan}

The original exit plan for this LDRD was to use our results to leverage funding from DHS Advanced Scientific Computing based on modeling interest from the Biobriefcase team, an LLNL DHS-funded device project. A simulation for DNA extraction in a 2D representation of a pillar chip was successfully performed as proof-of-concept $[6,7]$. At the beginning of our last year of funding, DHS ASC decided to no longer fund simulation work. We then focused our attention on followon funding from $\mathrm{NIH}$ because our work is also applicable to the design of biomedical microdevices as well as cardio-pulmonary flows; both are important to the healthcare industry. We have several proposals pending. We are also seeking to continue basic applied math research of complex flows through the DOE Office of Science Applied Math Base Program. Since this work leveraged the Chombo software developed under the DOE SciDAC program some aspects of this project are ongoing. Our capability continues to attract interest from MEMS-based device projects at LLNL.

\section{Summary}

The result of this LDRD project is an LLNL capability to model complex biological fluids like DNA at the microscale for the purpose of designing and optimizing microdevice components. We began this project intending to model a range of physical phenomena observed in microfluidic devices in order to provide a generalpurpose computational tool for device development. For relevance to device projects at LLNL we concluded this work by focusing on the design needs of the Biobriefcase, specifically, DNA extraction chambers in the PCR module $[5,6]$. 
This work has led to several milestones notable in the scientific community. The first is the development of an algorithm for continuum viscoelasticity which solves the High Weissenberg Number Problem, a decades old problem in the non-Newtonian flow community [29]. Though our interest was to use Oldroyd-B as a model for long-chain polymers (like DNA) in a fluid at the microscale, because of continuum scaling, our viscoelastic model and algorithm are also applicable to macroscale industrial flows such as polymer extrusion. A second scientific achievement is the development of a fully coupled fluid-polymer algorithm which promises to demonstrate for the first time the effect of a semi-dilute or concentrated solution of discretized particles on a fluid [7]. In this hybrid algorithm we have also explored and modeled short-range interactions between polymers, as well as electrokinetic effects. Finally, our methodology for treating irregular geometry has been coupled to a fast and accurate technique for extracting surface data from patient-specific medical images without loss in anatomical detail [3].

\section{Acknowledgments}

The PI would like to thank his collaborators: Professor Greg Miller, at UC Davis for his outstanding effort in developing a state-of-the-art particle-fluid algorithm; Andy Nonaka, UC Davis, and Shelly Gulati, UC Berkeley, for validation of the computations with experiments; Professor Dorian Liepmann at UC Berkeley for providing the microfluidic device motivation; and Phil Colella and the Applied Numerical Algorithms Group at LBNL for their support with the Chombo software and algorithms. 


\section{Figures}

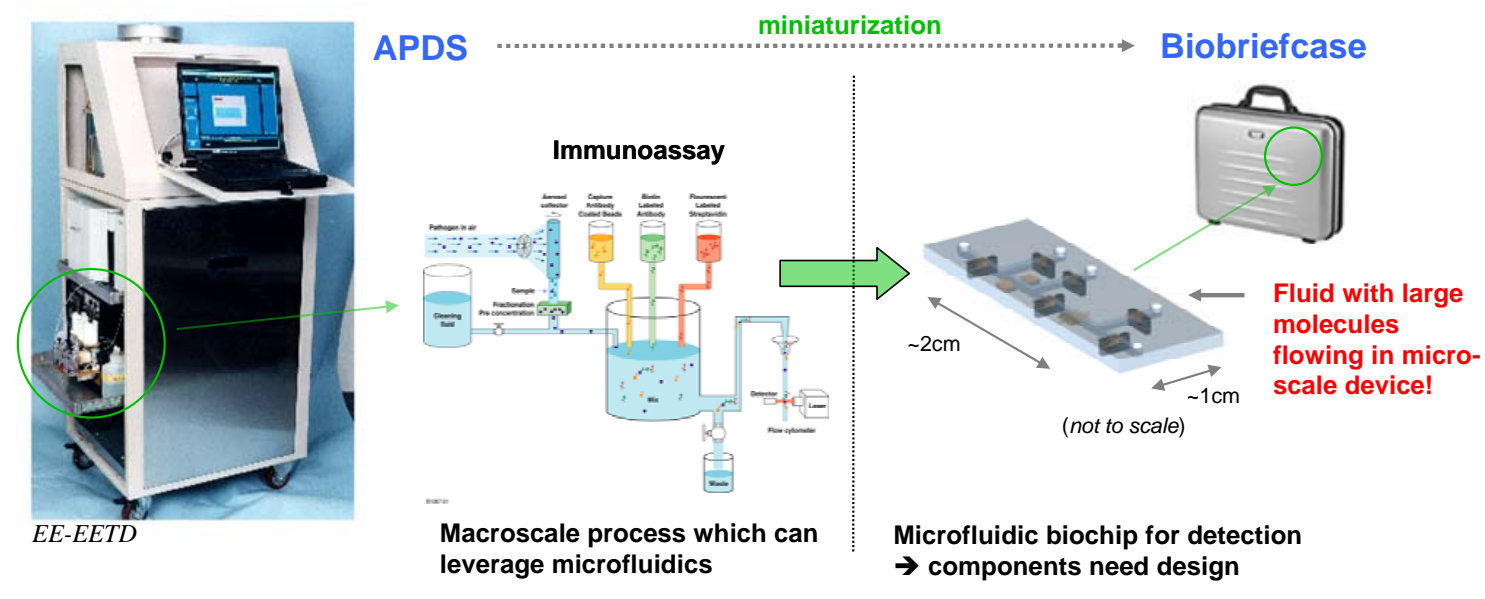

Figure 1 Miniaturization design strategy for Autonomous Pathogen Detection System (APDS). Macroscale biochemical detection process will leverage microfluidic technology and housed in a small briefcase size device.

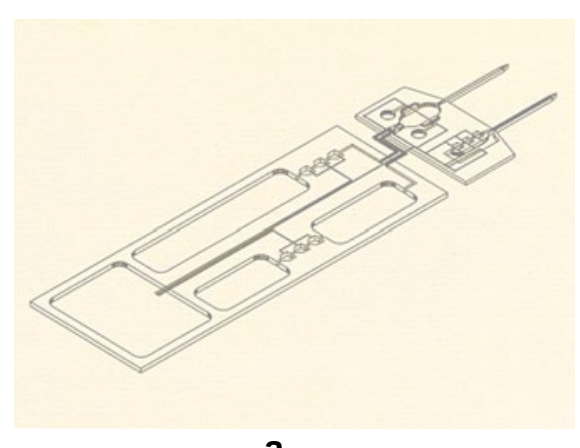

a

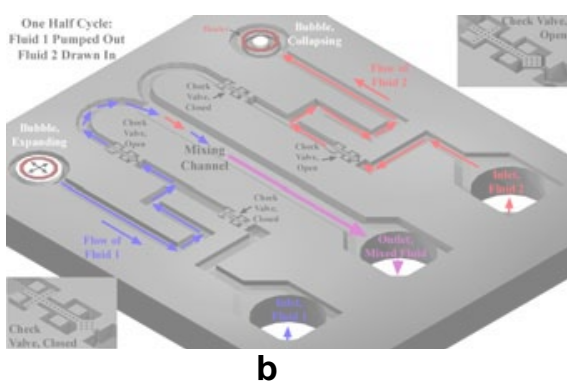

b

Figure 2 (a) Prototype integrated continuous monitoring and drug delivery system. (b) A working microfluidic mixer with canonical components developed under the DARPA $\mu$ FLUMES program. (Liepmann, UCB BioE) 


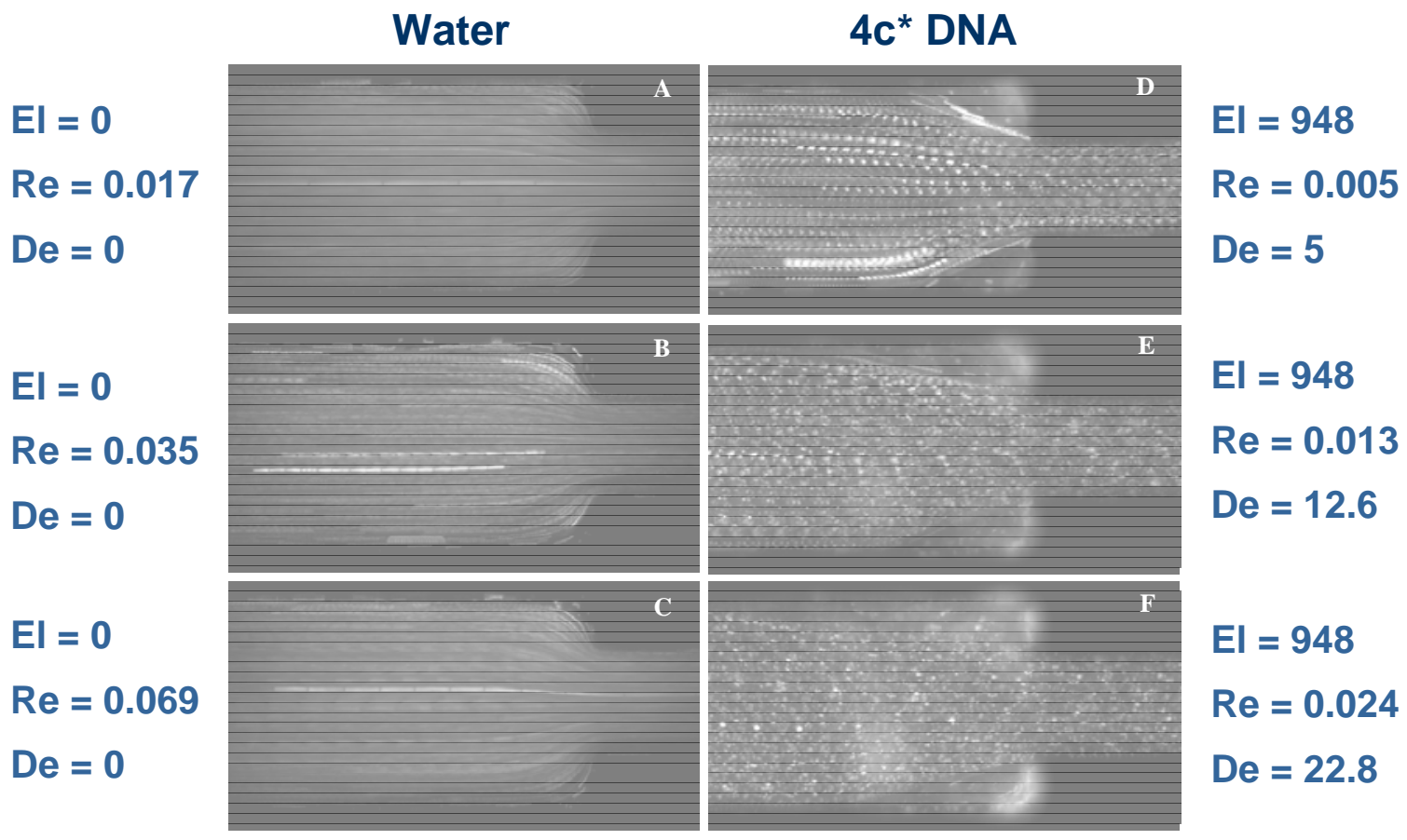

Figure 3 Continuum behavior of DNA in microscale flow. Streak images comparing flow of water (left) and DNA (right) in a sudden planar contraction for increasing elasticity. The viscoelastic behavior of this DNA solution is demonstrated by large, slowly recirculating vortices in the salient corners of image on the right. Flow is from left to right in each image. This data is a result of the project, and thus, proof of a hypothesis that DNA at microscale exhibits complex non-Newtonian behavior. Gulati, Muller, Liepmann and Trebotich

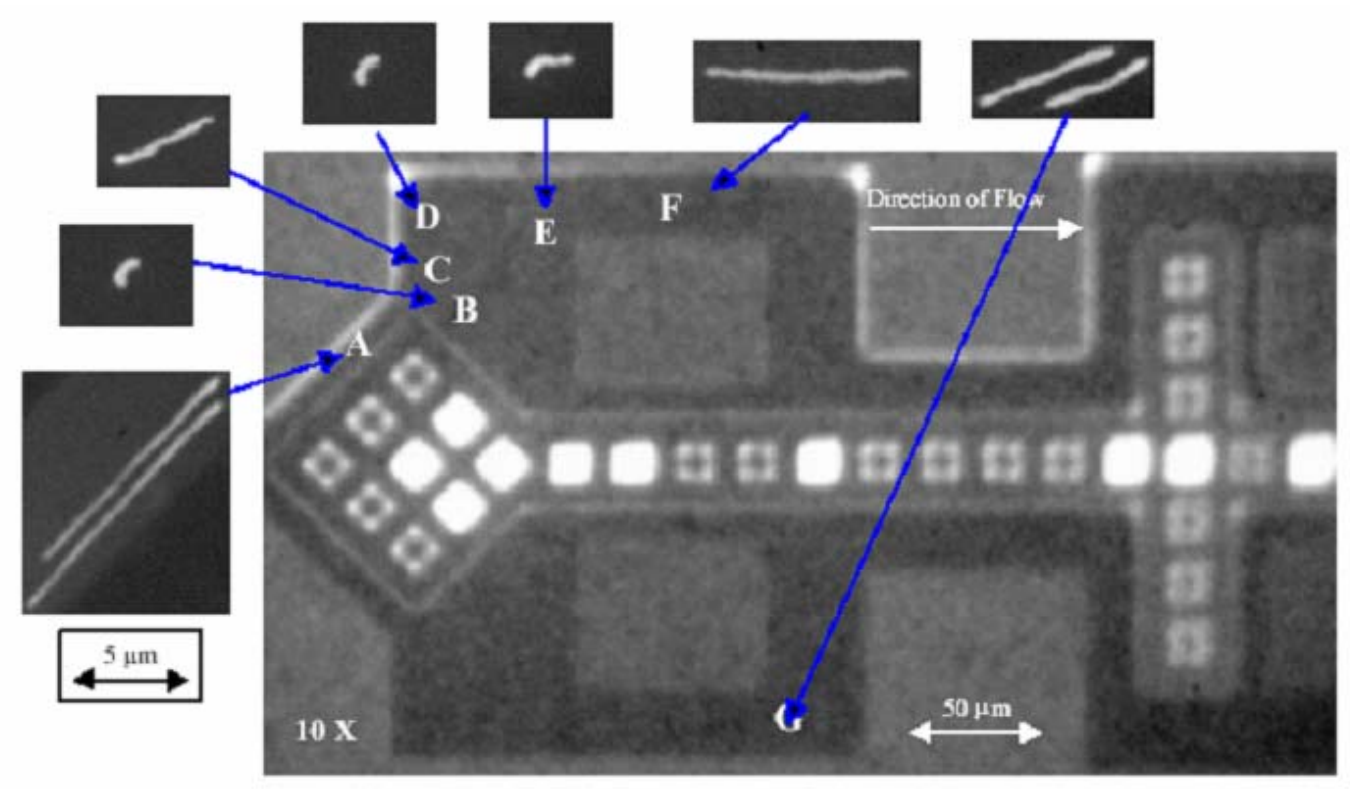

Figure 4 Variations in the conformation of DNA molecules traveling through a micro-check valve. The $5 \mathrm{~mm}$ length scale shown is consistent for all the DNA pictures. The molecules stretch out in the accelerating section of the flow and then recoil in regions of expansion flows [1]. 

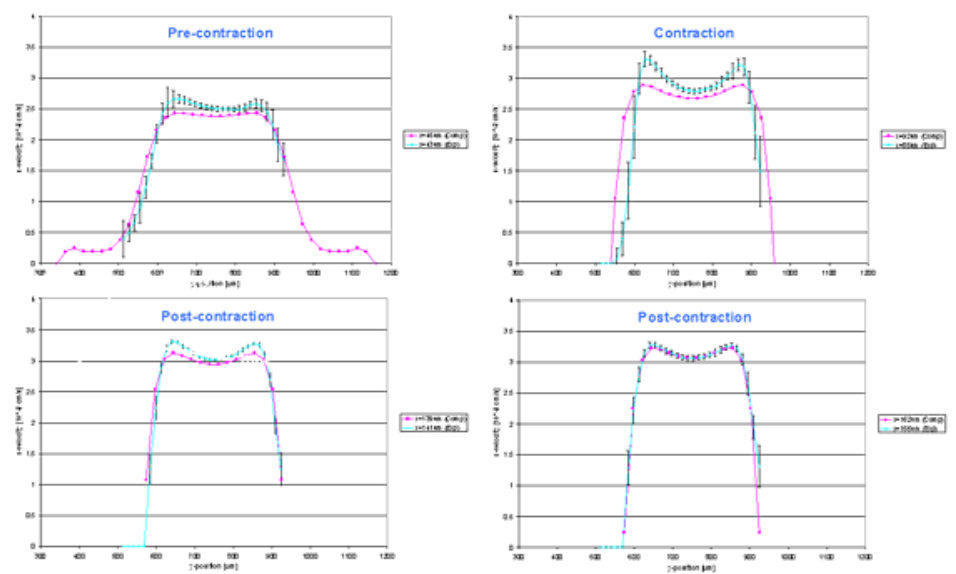

Figure 5 Preliminary low concentration validation. Computational versus experimental velocity profiles at various locations before, at and after sudden contraction in microchannel. Agreement is good, but off a bit due to model geometry being different from experiment, particularly near the contraction as in plot on top right.

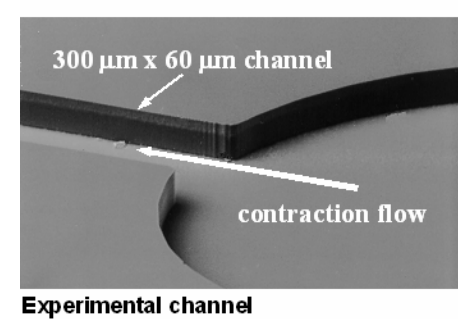

a

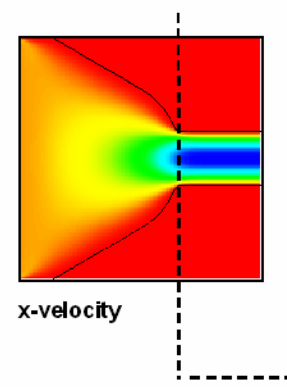

b

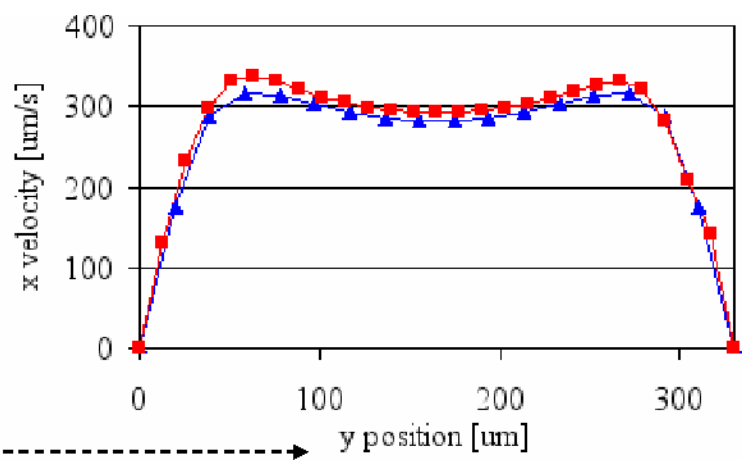

C

Figure 6 Improvement of low concentration validation. a) Experimental microchannel. (b) Horizontal velocity computed with viscoelastic model. (c) Comparison of experimental DPIV (red) and computational (blue) velocity profiles at contraction. [1] 


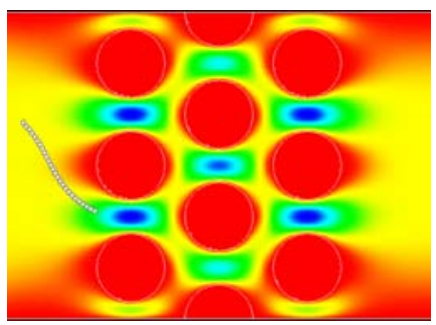

a

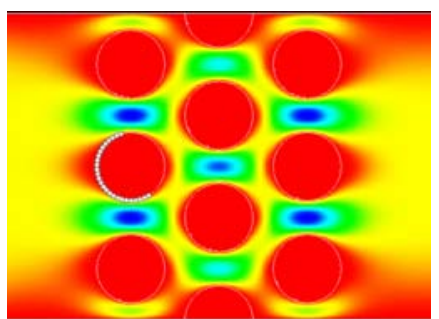

b

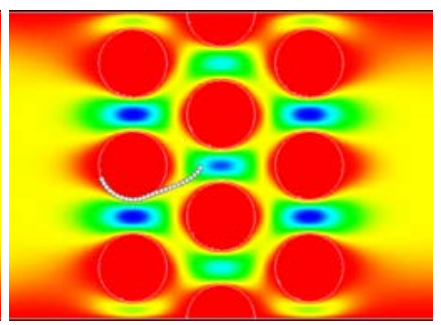

C
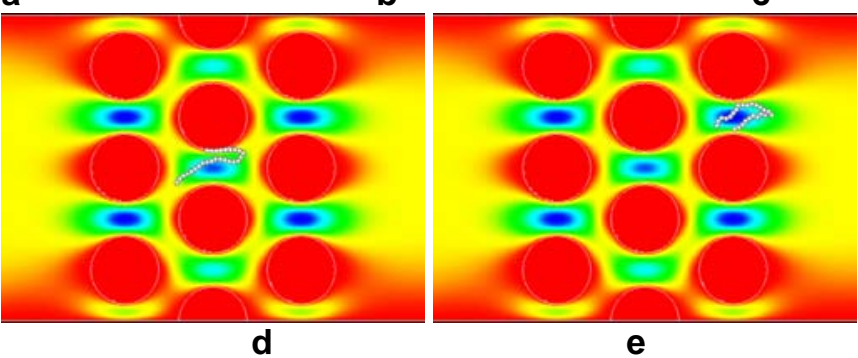

Figure 7 Time sequence of genomic DNA flowing in 2D model of pillar chip PCR chamber. No intra-polymer interaction (rods cross). DNA molecule enters from left in (a), then wraps around bead in (b), is loosened by hydrodynamic and Brownian forces in (c) and is swept out of the chamber by the flow field in (d) and (e). Color map indicates underlying flow field. [6, 7]

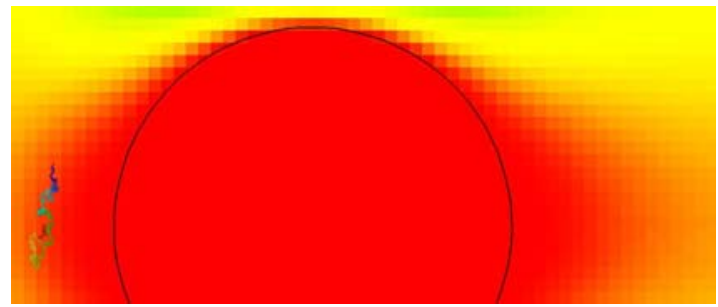

a

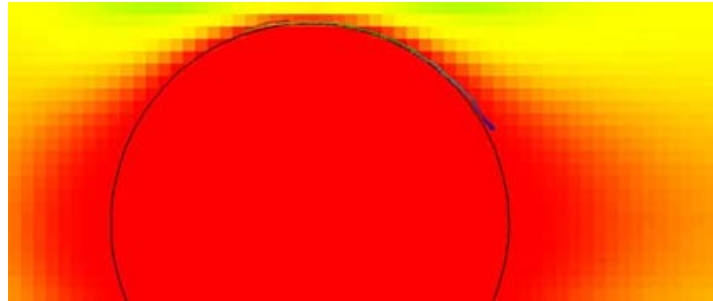

C

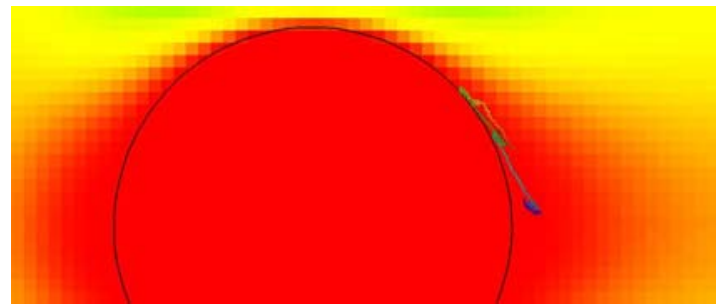

e

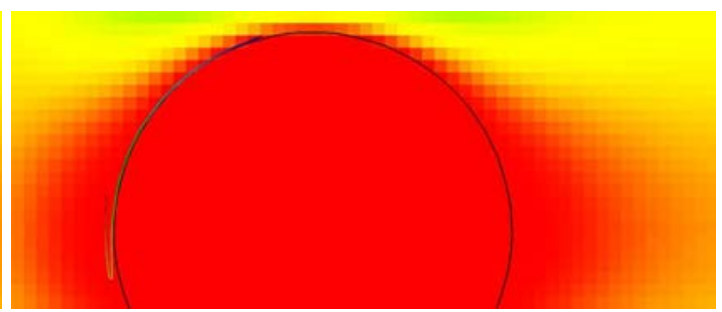

b

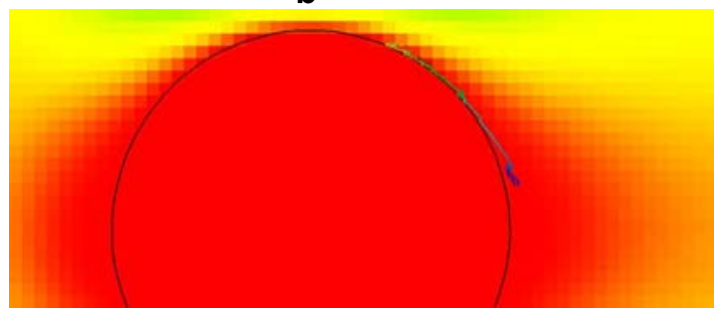

d

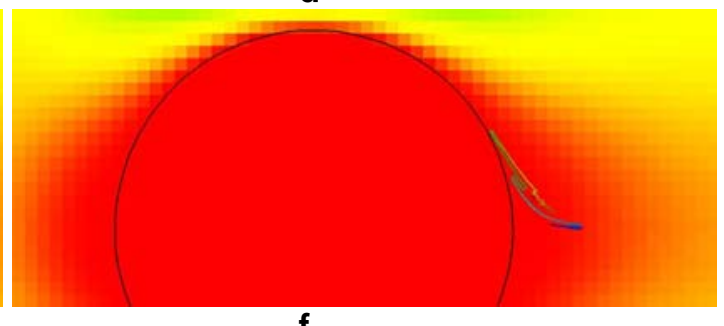

f

Figure 8 Time sequence of intra-polymer and polymer-structure interactions. (a) Nearly entangled polymer. (b) Short-range attachment. (c) Acceleration around pillar due to Brownian perturbation and hydrodynamic drag. (d) More acceleration of the tail and slowing of the head in stagnation region in wake of pillar. (e) Accelerated tail catching up with stagnated head. (f) Reentanglement in wake. [2] 


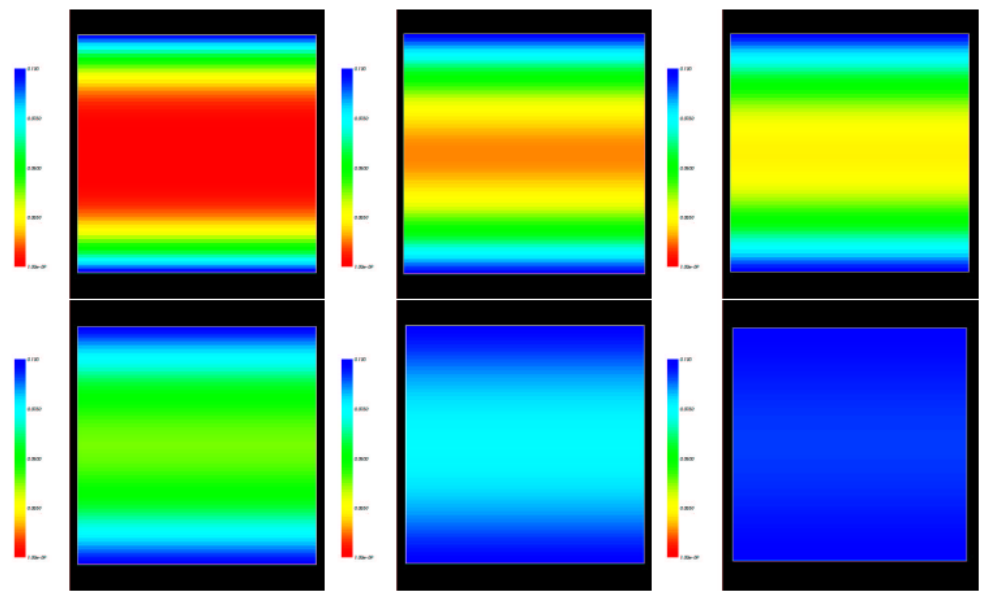

Figure 9 Fluid flow in a rectangular channel driven by an electrokinetic slip-velocity at the top and bottom boundaries at times $\mathrm{t}=0.00012 \mathrm{sec}, \mathrm{t}=0.00037 \mathrm{sec}, \mathrm{t}=0.00049 \mathrm{sec}$, $\mathrm{t}=0.00073 \mathrm{sec}, \mathrm{t}=0.00171 \mathrm{sec}$, and $\mathrm{t}=0.00281 \mathrm{sec}$ (ordered left to right, top to bottom). A potential drop of $1 \mathrm{~V}$ in the $\mathrm{x}$-direction is instantaneously switched on at $\mathrm{t}=0 \mathrm{sec}$. The channel is $100 \mu \mathrm{m}$ wide, the fluid is an aqueous solution with a kinematic viscosity of $0.01 \mathrm{~cm}^{2} / \mathrm{sec}$, and the electro-osmotic mobility is 0.001 . With this set of parameters, the steady-state plug flow should have a velocity of $0.1 \mathrm{~cm} / \mathrm{sec}$ which is essentially what is observed by time $\mathrm{t}=0.00281 \mathrm{sec}$. [5]

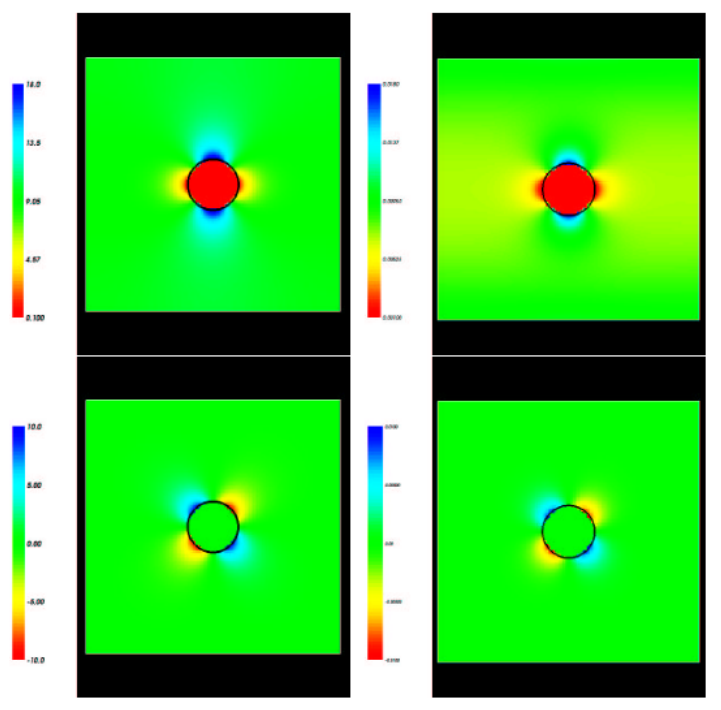

Figure 10 Comparison of electric field to steady fluid flow field in a rectangular channel containing a cylindrical post near steady-state $(\mathrm{t}=11.572 \mathrm{sec})$. The top row compares the $\mathrm{x}-$ component of the electric and velocity fields (electric field on left, velocity field on right); the bottom compares the y-components. Electrokinetic slip occurs at the top and bottom boundaries as well as on the surface of the post. A potential drop of $10 \mathrm{~V}$ in the $\mathrm{x}$-direction is instantaneously switched on at $\mathrm{t}=0 \mathrm{sec}$. The channel is $1 \mathrm{~cm}$ wide, the post has a radius of $1 \mathrm{~mm}$, the fluid is an aqueous solution with a kinematic viscosity of $0.01 \mathrm{~cm}^{2} / \mathrm{sec}$, and the electro-osmotic mobility on all physical boundaries is 0.001 . [5] 


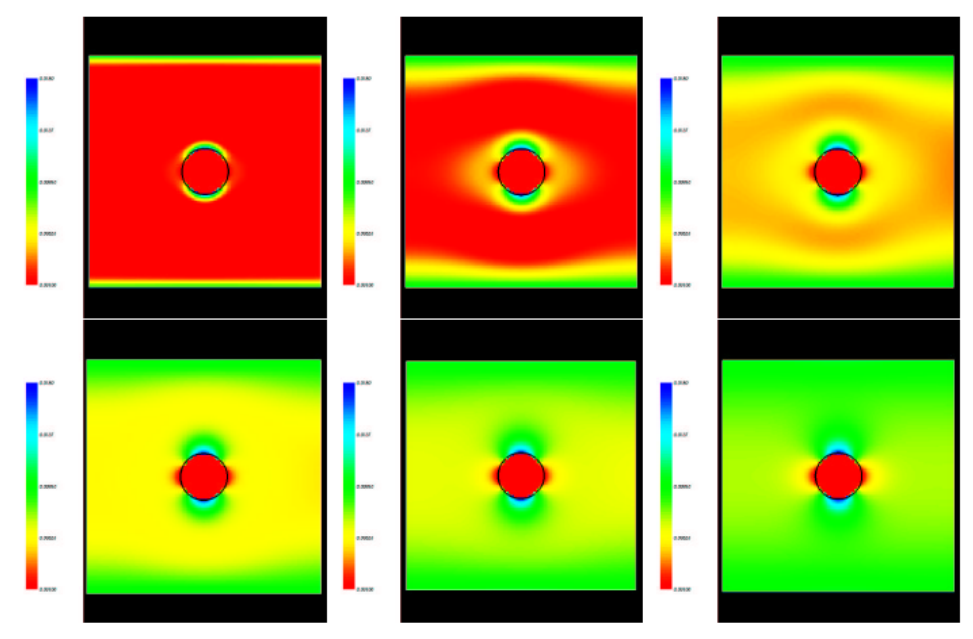

Figure $112 \mathrm{D}$ fluid flow in a rectangular channel containing a cylindrical post at times $\mathrm{t}=0.0610 \mathrm{sec}, \mathrm{t}=1.221 \mathrm{sec}, \mathrm{t}=4.272 \mathrm{sec}, \mathrm{t}=6.104 \mathrm{sec}, \mathrm{t}=8.545 \mathrm{sec}$, and $\mathrm{t}=11.572 \mathrm{sec}$ (ordered left to right, top to bottom). Electrokinetic slip occurs at the top and bottom boundaries as well as on the surface of the post. A potential drop of $10 \mathrm{~V}$ in the $\mathrm{x}$-direction is instantaneously switched on at $\mathrm{t}=0 \mathrm{sec}$. The channel is $1 \mathrm{~cm}$ wide, the post has a radius of $1 \mathrm{~mm}$, the fluid is an aqueous solution with a kinematic viscosity of $0.01 \mathrm{~cm}^{2} / \mathrm{sec}$, and the electro-osmotic mobility on all physical boundaries is 0.001 . [5]

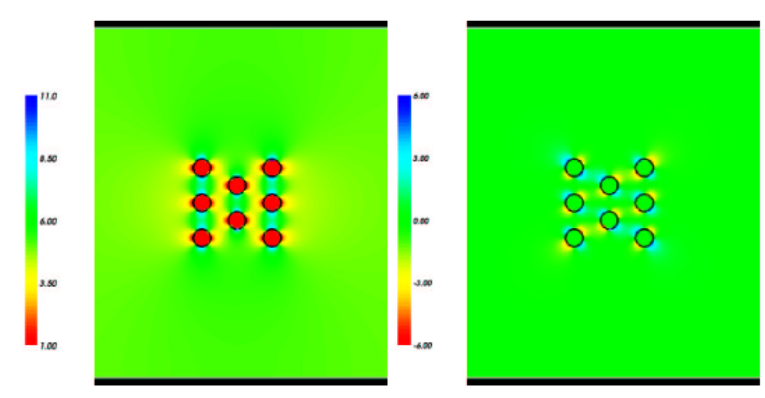

Figure 12 Electric field for a rectangular channel containing a staggered array of cylindrical posts ( $E_{x}$ on left, $E_{y}$ on right). Electrokinetic slip occurs at the top and bottom boundaries as well as on the surface of all posts. A potential drop of $1 \mathrm{~V}$ in the $\mathrm{x}$-direction is instantaneously switched on at $\mathrm{t}=0 \mathrm{sec}$. The channel is $2 \mathrm{~mm}$ wide, the each post has a radius of $25 \mu \mathrm{m}$, the fluid is an aqueous solution with a kinematic viscosity of $0.01 \mathrm{~cm}^{2} / \mathrm{sec}$, and the electro-osmotic mobility on all physical boundaries is 0.001 . [5] 


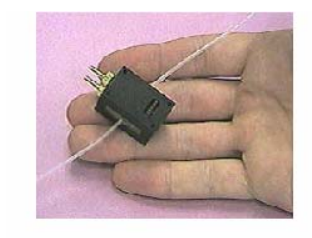

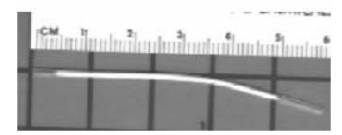

a

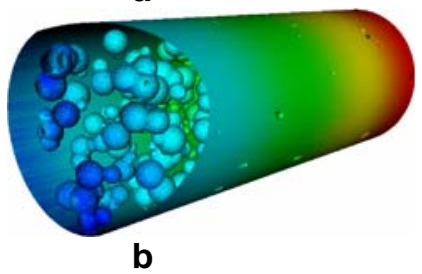

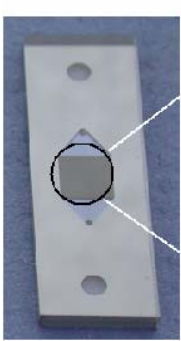

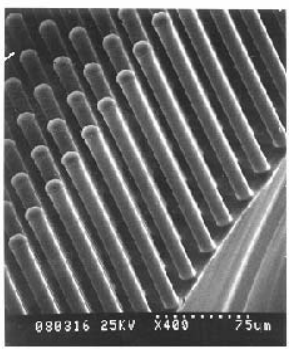

C

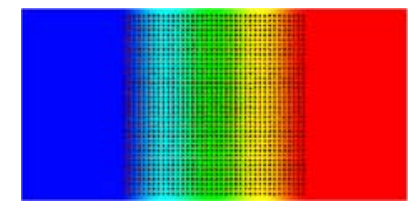

d

Figure 13 DNA extraction chambers: device and model. (a) Flow-through PCR thermal cycler (top) contains a packed bed DNA extraction chamber (external view at bottom) which is packed with $50 \mu \mathrm{m}$ beads. (b) Cut-away view of 3D continuum model for packed bed geometry with beads. (c) Pillar chip with SEM of array channel. (d) 2D continuum model (pressure data) for array channel. [6]

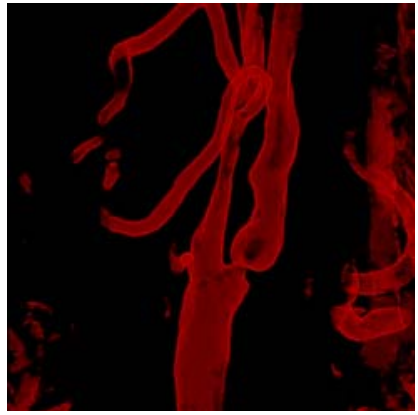

a

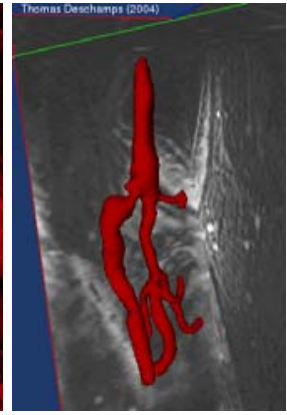

b

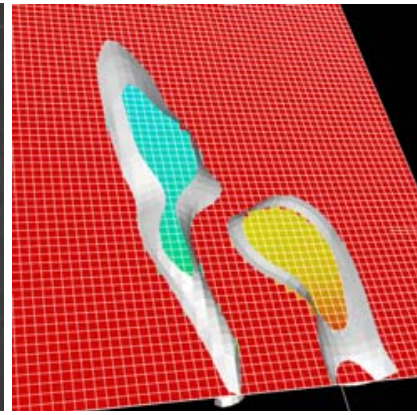

C

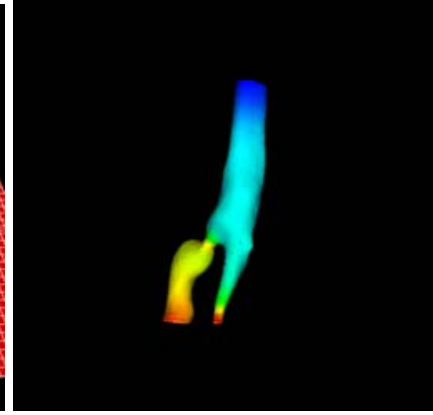

d

Figure 14 Patient-specific stenotic carotid artery: image to mesh to simulation. (a) Volume rendering from MRI. (b) Level-sets on image slice. (c) Detail of extracted surface intersecting Cartesian mesh (embedded boundary method). (d) 3D simulation of continuum flow representing pressure. [3]

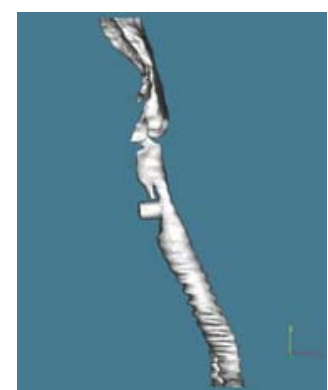

a

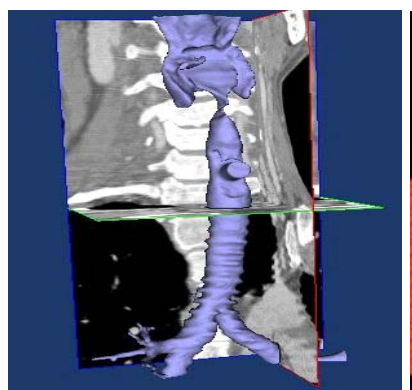

b

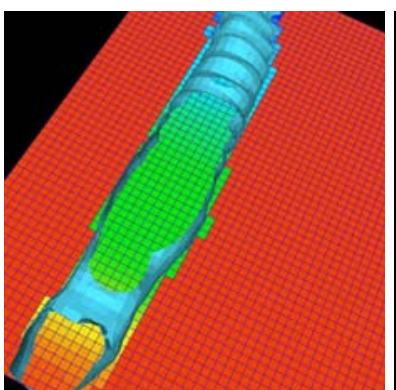

C

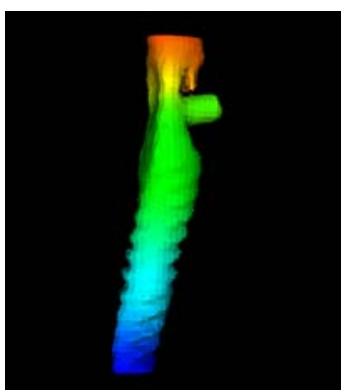

d

Figure 15 Patient-specific trachea with tracheostomy: image to mesh to simulation. (a) Volume rendering from CT. (b) Level-sets on image slice. (c) Detail of extracted surface intersecting Cartesian mesh (embedded boundary method). (d) 3D simulation of air-flow representing pressure. Note the geometric details in the rippled surface. [4]10 


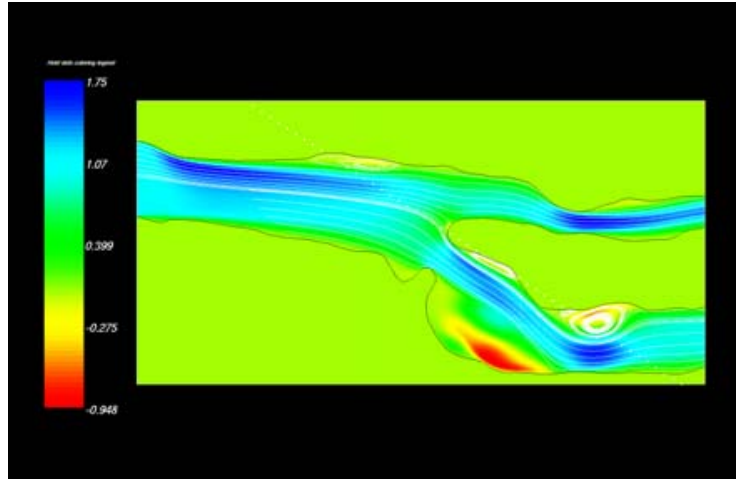

$\mathbf{a}$

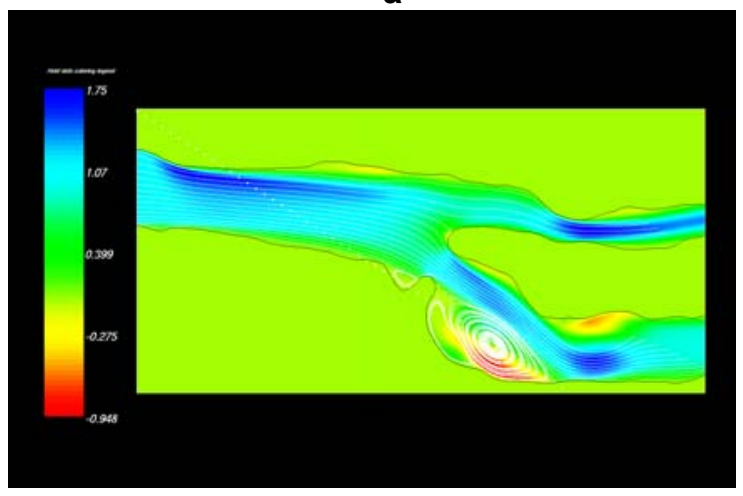

C

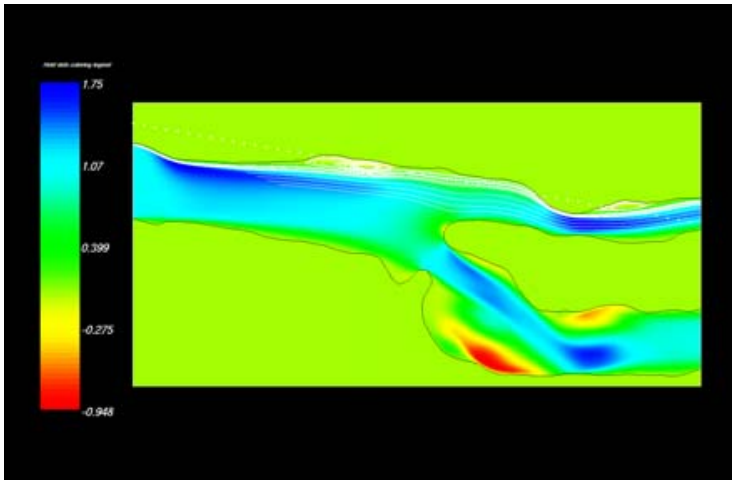

b

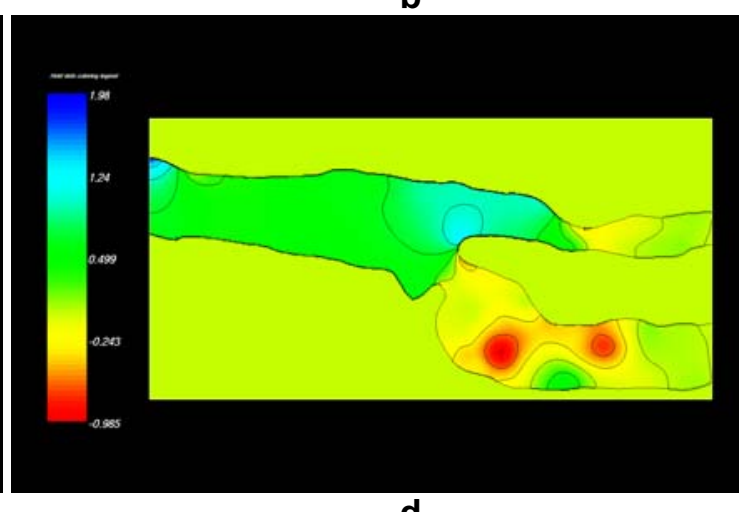

d

Figure 16 2D slices of patient-specific stenotic carotid artery. (a) Normal velocity with streamlines, middle seed points. (b) Normal velocity with streamlines, upper seed points. (c) Normal velocity with streamlines, lower seed points. (d) Pressure with contours.

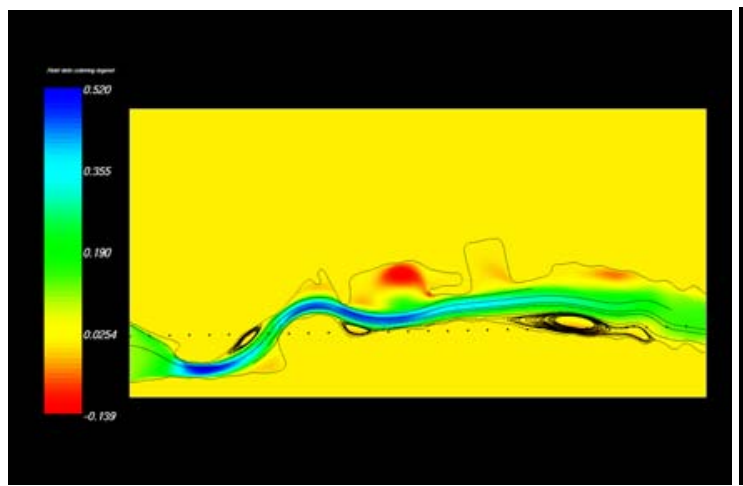

a

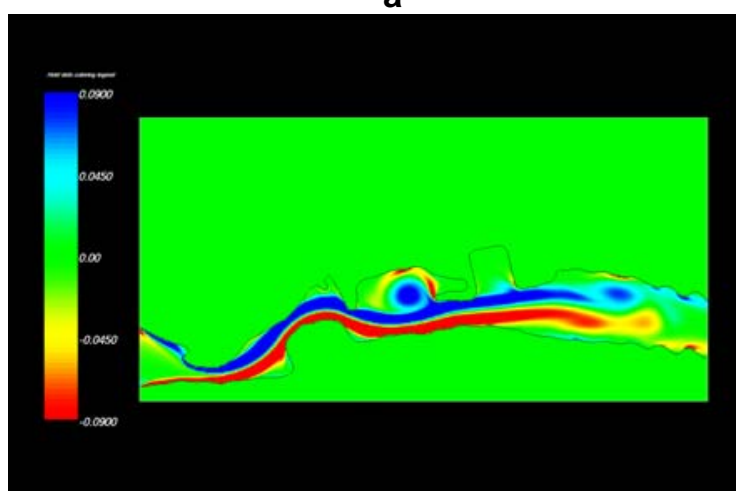

C

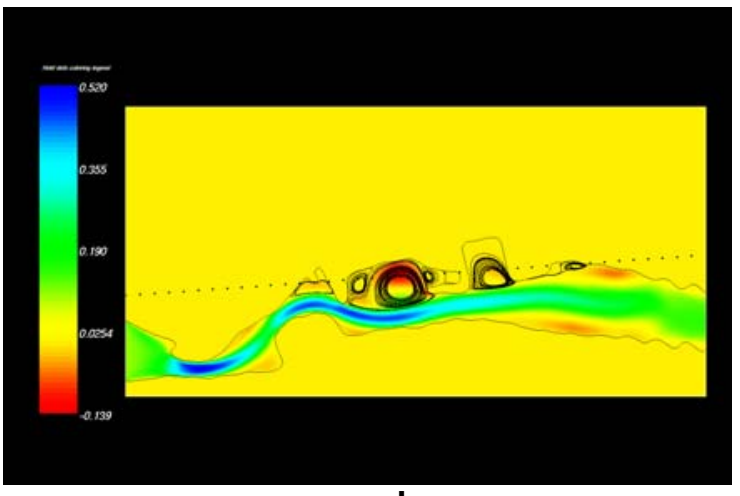

b

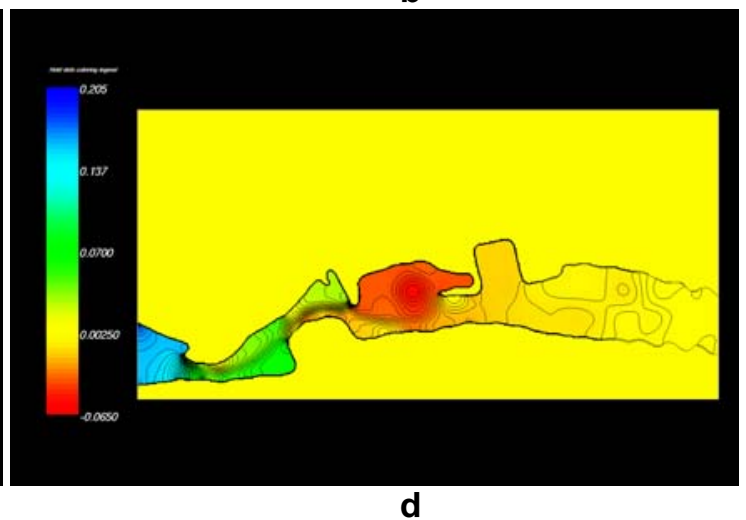

Figure 17 Patient-specific trachea with tracheostomy. (a) Normal velocity with streamlines, lower seed points. (b) Normal velocity with streamlines, upper seed points. (c) Vorticity. (d) Pressure with contours. 


\section{References}

1. Shrewsbury, P., Muller, S.J . and Liepmann, D., Concentration effects of a biopolymer in a microfluidic device. Biomed. Microdevices, 2001. 3: p. 225238.

2. Bybee, M.D., Miller, G. H., and Trebotich, D., Particle interactions in DNA flows. 2005, Lawrence Livermore National Laboratory technical report UCRLTR-217843.

3. Deschamps, T., Schwartz, P., Trebotich, D., Colella, P., Saloner, D. and Malladi, R., Vessel Segmentation and Blood Flow Simulation Using Level-Sets and Embedded Boundary Methods. International Congress Series, 2004. 1268: p. 75-80.

4. Deschamps, T., Schwartz, P. and Trebotich, D. Air-flow simulation in realistic models of the trachea. in 26th Annual International Conference IEEE Engineering in Medicine and Biology Society. 2004. San Francisco, CA.

5. Chu, K., and Trebotich, D., Incorporating electrokinetic phenomena into an embedded boundary, Navier-Stokes code. 2005, Lawrence Livermore National Laboratory technical report UCRL-TR-218083.

6. Trebotich, D., and Miller, G. H. Modeling and simulation of DNA flow in a microfluidic-based pathogen detection system. in 3rd Annual International IEEE-EMBS Special Topic Conference on Microtechnologies in Medicine and Biology. 2005. Oahu, Hawaii.

7. Trebotich, D., Miller, G.H., Colella, P., Graves, D.T., Martin, D.F. and Schwartz, P.O., A Tightly Coupled Particle-Fluid Model For DNA Laden Flows in Complex Microscale Geometries. Computational Fluid and Solid Mechanics, 2005: p. 1018-1022.

8. Alves, M.A., Oliveira, P. J., and Pinho, F. T., Benchmark solutions for the flow of Oldroyd-B and PTT fluids in planar contractions. J. Non-Newtonian Fluid Mech., 2003. 110: p. 45-75.

9. Crochet, M.J., and Pilate, G., Plane flow of a second grade fluid through a contraction. J. Non-Newtonian Fluid Mech., 1976. 1: p. 247-158.

10. Crochet, M.J., Davies, A. R., and Walters, K., Numerical Simulation of NonNewtonian Flow. 1984, Amsterdam: Elsevier.

11. Keunings, R., On the High Weissenberg Number Problem. J. Non-Newtonian Fluid Mech., 1986. 20: p. 209-226.

12. Phillips, T.N., and Williams, A. J., Comparison of creeping and inertial flow of an Oldroyd-B fluid through planar and axisymmetric contractions. J. NonNewtonian Fluid Mech., 2002. 108: p. 25-47.

13. Boger, D.V., A highly elastic constant viscosity fluid. J. Non-Newtonian Fluid Mech., 1977. 3: p. 87-91.

14. Evans, R.E., and Walters, K., Flow characteristics associated with abrupt changes in geometry in the case of highly elastic liquids. J. Non-Newtonian Fluid Mech., 1986. 20: p. 11-29.

15. Evans, R.E., and Walters, K., Further remarks on the lip-vortex mechanism of vortex enhancement in planar-contraction flows. J. Non-Newtonian Fluid Mech., 1989. 32: p. 95-105.

16. Oldroyd, J.G., On the Formulation of Rheological Equations of State. Proc. Roy. Soc. London A, 1950. 200: p. 523-541.

17. Bell, J.B., Colella, P. and Glaz, H.M., A second-order projection method for the incompressible Navier-Stokes equations. J. Comput. Phys., 1989. 85: p. 257283.

18. Chorin, A.J., Numerical Solution of the Navier-Stokes Equations. Math. Comp., 1968. 22: p. 745-762. 
19. Hur, J.S., Shaqfeh, E. S. G and Larson, R. G, Brownian dynamics simulations of single DNA molecules in shear flow. J. Rheol., 2000. 44(4): p. 713-42.

20. Cortez, R., and Minion, M., The blob projection method for immersed boundary problems. J. Comput. Phys., 2000. 161: p. 428-453.

21. Johansen, H., and Colella, P., A Cartesian Grid Embedded Boundary Method for Poisson's Equation on Irregular Domains. J. Comput. Phys., 1998. 147(2): p. 60-85.

22. McCorquodale, P., Colella, P. and Johansen, H., A Cartesian grid embedded boundary method for the heat equation on irregular domains. J. Comput. Phys., 2001. 173: p. 620-35.

23. Modiano, D., and Colella, P. A higher-order embedded boundary method for time-dependent simulation of hyperbolic conservation laws. in FEDSM 00 ASME Fluids Engineering Simulation Meeting. 2000. Boston, MA.

24. Johansen, H., Cartesian Grid Embedded Boundary Methods for Elliptic and Parabolic Partial Differential Equations on Irregular Domains, in Mech. Eng. 1997, The University of California, Berkeley.

25. Aftosmis, M., Melton, J. and Berger, M., Robust and efficient Cartesian mesh generation for component-based geometry. AIAA J ournal, 1998. 36(6): p. 952-960.

26. Bazant, M.Z., and Squires, T.M., Induced-charge electro-kinetic phenomena: Theory and microfluidic applications. Phys. Rev. Lett., 2004. 92(6).

27. Trebotich, D., Colella, P., Miller, G. and Liepmann, D. A Numerical Model of Viscoelastic Flow in Microchannels. in 2003 Nanotechnology Conference and Trade Show. 2003.

28. Trebotich, D., Colella, P., Miller, G. H., Nonaka, A., Marshall, T., Gulati, S. and Liepmann, D. A Numerical Algorithm for Complex Biological Flow in Irregular Microdevice Geometries. in 2004 Nanotechnology Conference and Trade Show. 2004.

29. Trebotich, D., Colella, P. and Miller, G.H., A stable and convergent scheme for viscoelastic flow in contraction channels. J. Comput. Phys., 2005. 205: p. 315-342.

30. Nonaka, A., Gulati, S., Trebotich, D., Miller, G. H., Muller, S. J. and Liepmann, D. A Computational Model with Experimental Validation for DNA Flow in Microchannels. in 2005 NSTI Nanotechnology Conference and Trade Show. 2005.

31. Renardy, M., The stresses of an upper convected Maxwell fluid in a Newtonian velocity field near a re-entrant corner. J. Non-Newtonian Fluid Mech., 1993.

50: p. 127-134.

32. Trebotich, D., A stable and convergent scheme for viscoelastic flows in contraction channels. 2005, Lawrence Livermore National Laboratory technical report UCRL-PRES-211385.

33. Kramers, H.A., The behavior of macromolecules in inhomogeneous flow. J. Comput. Phys., 1946. 14: p. 415-24.

34. Somasi, M., Khomami, B., Woo, N.J., Hur, J.S. and Shaqfeh, E.S.G., Brownian dynamics simulation of bead-rod and bead-spring chains: numerical algorithms and coarse-graining issues. J. Non-Newtonian Fluid Mech., 2002. 108: p. 227-255.

35. Mazars, M., Freely jointed chains in external potentials: analytical computations. J. Phys. A: Math. Gen., 1999. 32: p. 1841-61. 